\title{
Inhibition of breast cancer growth in vivo by antiangiogenesis gene therapy with adenovirus- mediated antisense-VEGF
}

\author{
S-A Im¹, J-S Kim², C Gomez-Manzano ${ }^{3}$, J Fueyo ${ }^{3}$, T-J Liư ${ }^{3}$, M-S Cho ${ }^{4}$ C-M Seong ${ }^{1}$, SN Lee', Y-K Hong ${ }^{5}$ and WKA \\ Yung $^{3}$ \\ Departments of ${ }^{1}$ Internal Medicine and ${ }^{4}$ Pathology, Ewha Medical Research Center, Ewha Womans University; Departments of ${ }^{2}$ General Surgery and \\ ${ }^{5}$ Neurosurgery, Catholic University of Korea, Seoul, Korea; ${ }^{3}$ Department of Neuro-Oncology, The University of Texas M.D. Anderson Cancer Center, Houston, \\ TX, USA
}

Summary Increased expression of VEGF in several types of tumours has been shown to correlate with poor prognosis. We used a replication-deficient adenoviral vector containing antisense VEGF cDNA (Ad5CMV- $\alpha$ VEGF) to down-regulate VEGF expression and increase the efficiency of delivery of the antisense sequence in the human breast cancer cell line MDA231-MB. Transfection of these cells with Ad5CMV- $\alpha$ VEGF in vitro reduced secreted levels of VEGF protein without affecting cell growth. Moreover, injection of the Ad5CMV- $\alpha$ VEGF vector into intramammary xenografts of these cells established in nude mice inhibited tumour growth and reduced the amount of VEGF protein and the density of microvessels in those tumours relative to tumours treated with the control vector Ad5(dl312). Our results showed

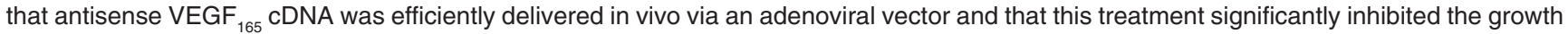
of established experimental breast tumours. The Ad5CMV- $\alpha$ VEGF vector may be useful in targeting the tumour vasculature in the treatment of breast cancer. (C) 2001 Cancer Research Campaign http://www.bjcancer.com

Keywords: breast cancer; antiangiogenesis; gene therapy; adenovirus; VEGF

The growth of solid tumours and the formation of metastases depend on the formation of new blood vessels (Folkman, 1994). Angiogenesis is a complex multistep process involving extracellular matrix remodelling, endothelial cell migration and proliferation, and capillary differentiation and anastomosis, all of which are regulated by angiogenic peptides (Blood and Zetter, 1990). Vascular density has been shown to be an independent prognostic marker in several types of human tumours, including breast carcinoma (Weidner et al, 1992; Im et al, 1997). Vascular endothelial growth factor (VEGF, also known as vascular permeability factor) is a strong endothelial cell mitogen that increases the permeability of microvessels (Dvorak et al, 1995). The effects of VEGF are mediated through 2 distinct high-affinity endothelial cell surface receptors, flt-1 and KDR/Flk-1, both of which are type III tyrosine kinase receptors (Shibuya, 1995). Increased expression of VEGF has been reported in several types of human tumours and has been shown to correlate with poor prognosis (Takahashi et al, 1995; Toi et al, 1995). In animal models, antisense VEGF and monoclonal antibody to VEGF inhibited VEGF expression and tumour growth (Kim et al, 1993; Asano et al, 1995; Cheng et al, 1996; Saleh et al, 1996).

Several reasons exist to suggest that gene therapy should be considered as a strategy to suppress the neovascularization of solid tumours. First, local, intratumoral gene therapy can reduce the risk of widespread antiangiogenesis resulting from systemic administration of an antiangiogenic agent. Second, gene transfer

Received 2 December 1999

Revised 7 August 2000

Accepted 20 November 2000

Correspondence to: S-A Im can lead to a local accumulation of the antiangiogenic protein. Third, current technology does not allow genes to be transferred to all of the target cells that are relevant to the growth of the tumour; however, antiangiogenic gene therapy does not require that genes be transferred to all target cells. Increasing knowledge of the molecular basis of cancer has yielded several potential therapies, but the clinical application of these methods has not been completely successful, in part because the in vitro models used for screening often do not duplicate in vivo conditions. To overcome this limitation, we developed an adenovirally mediated antisense-VEGF vector, Ad5CMV- $\alpha$ VEGF, that could be used to down-regulate VEGF secretion and thereby inhibit tumour growth in vivo. We previously found that transfecting the U87-MG glioma cell line with this vector down-regulated VEGF $_{165}$ mRNA and VEGF protein secretion compared with cells that had been transfected with Ad5(d1312) (Im et al, 1999). The purpose of the present study was to test whether treating MDA231-MB human breast cancer xenografts with the Ad5CMV- $\alpha$ VEGF vector would reduce the expression of VEGF protein and suppress tumour formation through antiangiogenesis mechanism in an experimental model of breast cancer.

\section{MATERIALS AND METHODS}

\section{Construction and generation of the adenoviral vectors}

To down-regulate endogenous VEGF expression and enhance the in vivo applicability of the antisense VEGF strategy, we constructed a replication-deficient recombinant adenoviral vector 
containing the cDNA for $\mathrm{VEGF}_{165}$ in an antisense orientation (Figure 1) as described previously (Zhang et al, 1993). Briefly, the 574-bp VEGF cDNA was cloned by using the pCRII vector (Invitrogen, Carlsbad, CA) and sequenced by using the $\mathrm{T} 7$ promoter and M13 reverse primers. Then, the VEGF cDNA was extracted from the pCRII vector with HindIII and Not I restriction enzymes and inserted in an antisense orientation into the E1deleted expression plasmid pXCL-cytomegalovirus (CMV) shuttle vector (a generous gift from Dr WW Zhang, Urogen Corp, San Diego, California, USA) between the CMV promoter and SV40 polyadenylation signal site to create the expression plasmid pXCL-CMV- $\alpha$ VEGF. This plasmid was cotransfected with plasmid PJM17 into the transformed human embryonic kidney cell line 293 (American Type Culture Collection, Manassas, VA) by the calcium phosphate method. Homologous recombination of the expression plasmid and pJM17 in 293 cells replaced the E1 region with the expression cassette from the expression plasmid. Then individual viral plaques were isolated, and plaques containing the human $\mathrm{VEGF}_{165}$ CDNA were identified by polymerase chain reaction (PCR) and restriction enzyme digestion and then amplified in 293 cells. Ad5(d1312), an E1-deleted adenovirus type 5 carrying no exogenous gene, was used as an adenoviral control vector (Jones and Shenk, 1979). Ad5CMV- $\alpha$ VEGF, Ad5(dl312), and Ad5CMV-ßgal were propagated in 293 cells and then purified on a cesium chloride gradient. The preparations were then dialysed and stored in dialysis buffer $\left(10 \mathrm{mmolL}^{-1}\right.$ Tris- $\mathrm{HCl}$ and $1 \mathrm{mmol}^{-1}$ $\mathrm{MgCl}_{2}, \mathrm{pH} 7.4$ ) with $10 \%$ glycerol at $-80^{\circ} \mathrm{C}$. The titre of each viral stock was determined in 293 cells by plaque assay (Graham and Prevec, 1991).

\section{Infection conditions}

Cell lines were infected as described previously (Gomez-Manzano et al, 1996). Briefly, cells were infected by diluting the viral stock to a specified concentration, adding the dilution to cell monolayers, and incubating the cells at $37^{\circ} \mathrm{C}$ for $30 \mathrm{~min}$ with brief agitation every $5 \mathrm{~min}$. After this, culture medium containing $10 \%$ serum was added, and the infected cells were returned to the $37^{\circ} \mathrm{C}$ incubator. Control cells were infected with the $\mathrm{Ad} 5(\mathrm{~d} 1312)$ or mock-infected with culture medium. Ad5CMV-ßgal, an adenovirus type 5-based vector that lacks E1 and contains the CMV promoter that drives the Escherichia coli LacZ gene coding for the $\beta$-galactosidase protein, was used to verify the efficiency of infection.

\section{Cell line and tissue culture conditions}

The human breast cancer cell line MDA231-MB was obtained from the American Type Culture Collection. MDA231-MB cells were maintained in DMEM/F-12 medium (1:1, vol:vol) supplemented with $10 \%$ fetal bovine serum in a humidified atmosphere containing $5 \% \mathrm{CO}_{2}$ at $37^{\circ} \mathrm{C}$. The 293 cells were maintained in high-glucose DMEM with $10 \%$ heat-inactivated fetal bovine serum.

\section{Transduction efficiency}

To verify the transduction efficiency of the Ad5 vector, we infected MDA231-MB cells $\left(10^{5}\right.$ cells per well) with Ad5CMV$\beta$ gal at multiplicities of infection (MOI) (the ratio of the number of infectious virions to the number of susceptible cells) ranging from
25 to 200.48 hours later, cells were fixed with $4 \%$ paraformaldehyde in a phosphate-buffered $\mathrm{NaCl}$ solution, and then stained with $1 \mathrm{mg} \mathrm{ml}^{-1} 5$-bromo-4-chloro-3-indolyl- $\beta$-D-galactopyranoside in 5 $\mathrm{mM}$ potassium ferricyanide, $5 \mathrm{mM}$ potassium ferrocyanide, and 2 $\mathrm{mM} \mathrm{MgCl}_{2}$ in phosphate-buffered $\mathrm{NaCl}$ solution at $37^{\circ} \mathrm{C}$ overnight. The efficiency of infection (the percentage of $\beta$-galactosidase-positive cells) was determined by the number of blue cells among 500 cells on triplicate dishes.

\section{Enzyme-linked immunosorbent assay (ELISA)}

To quantify human secretory VEGF VE5 $_{165}$ in conditioned medium, we used ELISA according to the manufacturer's protocol ( $R$ \& D Systems, Minneapolis, MN). To prepare the conditioned medium, cells were seeded overnight $\left(10^{5}\right.$ cells per well $)$ with medium containing $10 \%$ serum and then infected with either Ad5CMV- $\alpha$ VEGF or Ad5(d1312) at different MOI. Culture medium was used for the mock-infection condition. Triplicate dishes of cells subjected to each treatment were used. Conditioned media were processed 5 days after infection as follows. 30 hours before media collection, the cells were washed 3 times with $2 \mathrm{ml}$ of serum-free medium and then incubated with $2 \mathrm{ml}$ of serum-free medium per well for $6 \mathrm{~h}$, after which the medium was aspirated and the cells were washed again with $2 \mathrm{ml}$ of serum-free medium per well and incubated for 24 hours in $1 \mathrm{ml}$ of medium containing $2 \%$ serum. The medium was collected in a tube containing $1 \mu \mathrm{l}$ of $100 \mathrm{mM}$ phenylmethylsulphonyl fluoride.

\section{Cell growth rate in vitro}

MDA231-MB cells were seeded at a density of $10^{4}$ cells per well in six-well culture plates and allowed to adhere overnight. The next day, the cells were infected with 100 MOI of Ad5CMV$\alpha$ VEGF or Ad5(d1312) or with culture medium. Triplicate dishes of each treatment were counted at regular intervals until the 12th day after infection.

\section{Ad5CMV- $\alpha$ VEGF treatment in vivo}

Animal experiments were carried out in the animal facility of The University of Texas MD Anderson Cancer Center in accordance with institutional guidelines. For these experiments, athymic female nu/nu mice, 4-6 weeks of age, were acclimated and caged in groups of 5 or fewer. All mice were fed a diet of animal chow and water ad libitum. The animals were anaesthetized with methoxyflurane before all procedures and were observed until full recovery. To create xenograft tumours, MDA231-MB cells $\left(5 \times 10^{6}\right.$ in $100 \mu \mathrm{l}$ of serum-free medium) were injected s.c. into the mammary fat pads of the mice. Beginning 4 days after tumour-cell implantation, tumours were treated by intratumoral injection of $5 \times 10^{8}$ plaqueforming units (PFU) of either Ad5(d1312) or Ad5CMV- $\alpha$ VEGF (8 mice/group) every other day for a total of 4 times; 3 of these 4-dose treatment cycles were conducted at 4 -week intervals. Tumour volume was calculated from weekly caliper measurements of the largest (a) and smallest (b) diameters of each tumour using the formula $a \times b^{2} \times 0.4$ (Attia and Weiss, 1966).

\section{Immunohistochemical analysis and microvessel counting}

At the end of the 130-day measurement period, the mice were killed 
with $\mathrm{CO}_{2}$, and their tumours were excised and fixed in neutralbuffered formalin for routine histologic examination and immunohistochemical staining. Tumours were analysed with an anti-VEGF $\mathrm{mAb}$ (PharMingen, San Diego, CA) to see the expression of VEGF and with an anti-CD31 mouse mAb (Biogenix, San Ramon, CA) to count microvessels as follows. Each paraffin block was serially sectioned at $4 \mu \mathrm{m}$ intervals, dewaxed, and heated in a microwave for $10 \mathrm{~min}$ to retrieve antigen. Endogenous peroxidase in the histologic sections was removed by incubation with $3 \%$ hydrogen peroxide in water for $15 \mathrm{~min}$ at room temperature. The primary antibodies were diluted 1:200 (VEGF) or 1:10 (anti-CD31) in phosphate-buffered saline with $0.5 \%$ bovine serum albumin, and the dilutions were added to the sections and incubated either for $1 \mathrm{~h}$ at room temperature (VEGF) or overnight (CD31). The bound antibodies were detected by the avidin-biotin-peroxidase method and visualized with 3-amino-9-ethylcarbazole (AEC). Cellular nuclei were counterstained blue with haematoxylin.

The areas containing the greatest numbers of microvessels or tumour 'hot spots' were identified by scanning the stained sections at low magnification $(40 \times$ and $100 \times)$ with a light microscope. Once these areas were identified, individual stained microvessels were point-counted at $200 \times$ magnification with a square grid that corresponded to a field size of $0.68 \mathrm{~mm}^{2}$. Large microvessels and any single brown-stained endothelial cells that were clearly separate from other microvessels were included in the microvessel count; branching structures were counted as 1 vessel unless there was a break in the continuity of the vessel, in which case it was counted as 2 distinct vessels. The mean number of microvessels from 3 fields was used for analysis.

\section{Statistical analysis}

All values were expressed as means \pm standard deviations. Unpaired $t$-tests were used to evaluate differences in tumour volumes in the different treatment groups. A $P$ value of less than 0.05 was considered to be statistically significant.

\section{RESULTS}

\section{Adenovirus-mediated gene transfer in MDA231-MB cells}

We assessed the efficiency of gene transfer via the replication-defi-

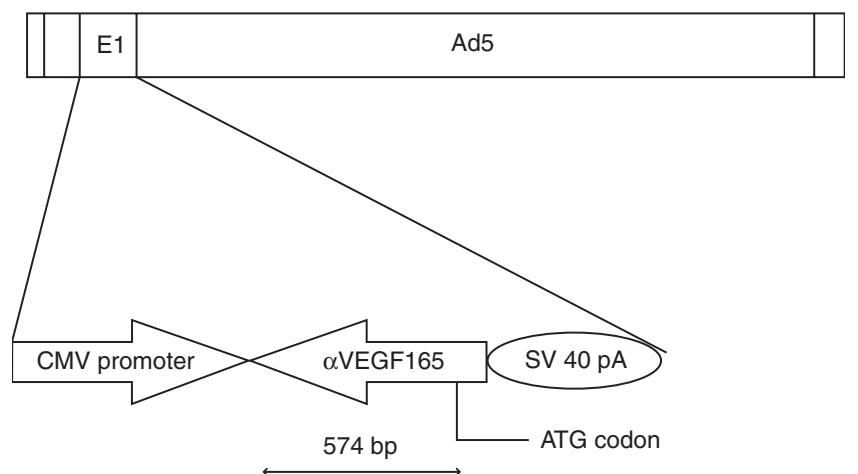

Figure 1 Structure of the recombinant adenoviral vector Ad5CMV- $\alpha$ VEGF The entire vector is shown at top, and an enlargement of human antisenseVEGF expression cassette is shown at bottom. The expression cassette

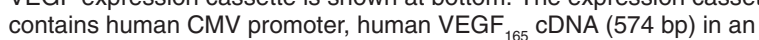
antisense orientation, and the SV40 polyadenylation (pA) signal

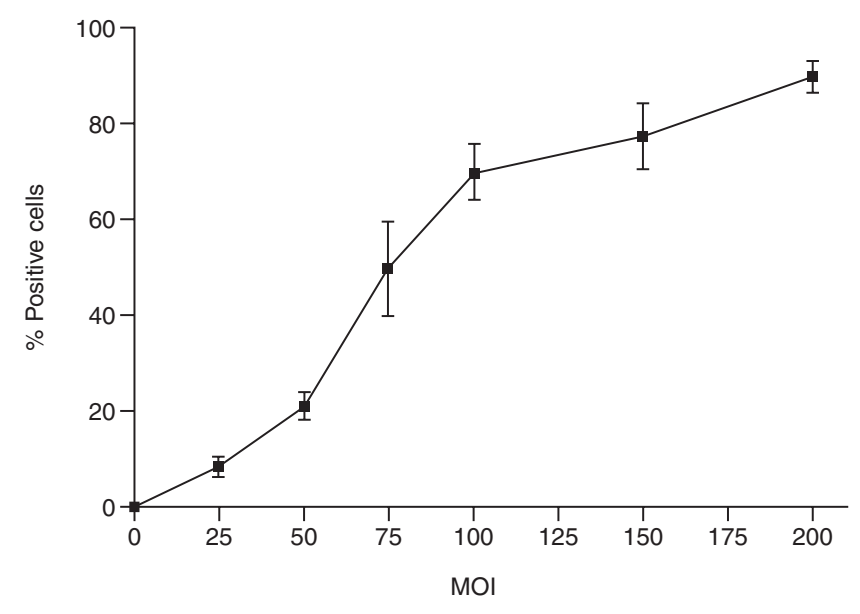

Figure 2 Ad5CMV-ßgal transduction efficiency in MDA231-MB breast cancer cells. Cells were infected with Ad5CMV- $\beta$ gal at the MOI values shown. The percentage of blue cells were determined by counting the number of blue cells among 500 cells on each of 2 dishes, then dividing this number by 500 and multiplying by 100 . The results shown are the means from triplicate experiments

cient recombinant adenoviral vector Ad5CMV- $\alpha$ VEGF, which contains the cDNA for $\mathrm{VEGF}_{165}$ in an antisense orientation (Figure 1), in the MDA231-MB breast cancer cell line by measuring reporter gene expression $48 \mathrm{~h}$ after cells were infected with Ad5CMV- $\beta$ gal at different MOI. The transduction efficiency ranged from $8.3 \pm 2.05 \%$ at $25 \mathrm{MOI}$ to $89.4 \pm 3.30 \%$ at $200 \mathrm{MOI}$ (Figure 2).

\section{VEGF expression and cell growth after treatment with Ad5CMV- $\alpha$ VEGF in vitro}

We used ELISA to determine the amount of secretory VEGF protein in conditioned medium collected 5 days after MDA231-MB cells has been mock-infected or infected with Ad5(dl312) or Ad5CMV$\alpha$ VEGF. The mock-infected MDA231-MB cells secreted VEGF protein at a concentration of $110.98 \pm 13.63 \mathrm{pg} \mathrm{ml}^{-1} 10^{5} \mathrm{cells}^{-1} 24 \mathrm{~h}^{-1}$; cells infected with Ad5(d11312) produced $105.73 \pm 10.03 \mathrm{pg} \mathrm{ml}^{-1}$ $10^{5}$ cells $^{-1} 24 \mathrm{~h}^{-1}$. In contrast, cells infected with $100 \mathrm{MOI}$ of Ad5CMV- $\alpha$ VEGF produced $73.24 \pm 7.02 \mathrm{pg} \mathrm{ml}^{-1} 10^{5}$ cells $^{-1} 24 \mathrm{~h}^{-1}$

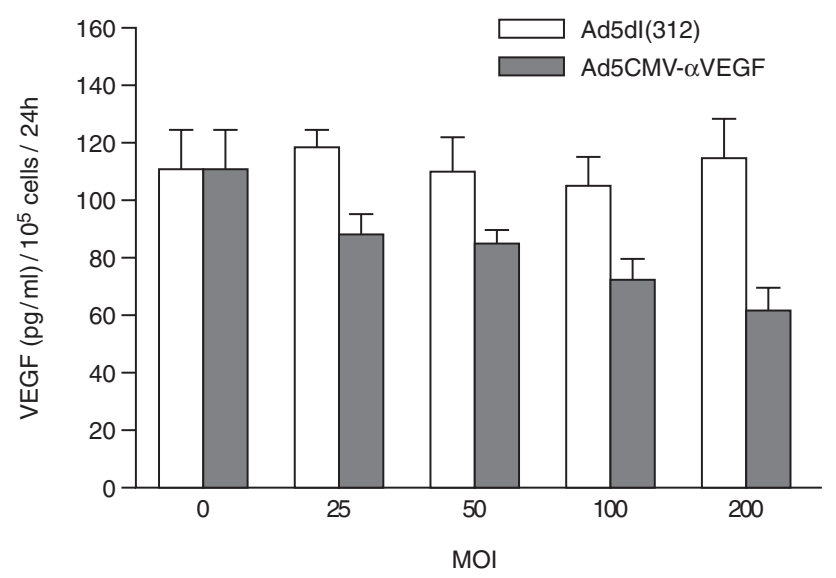

Figure 3 VEGF protein production. MDA231-MB cells were infected with Ad5(d1312) or Ad5CMV- $\alpha$ VEGF, and conditioned medium collected 5 days later was subjected to ELISA. Error bars indicate SD from 3 replicate experiments 
(Figure 3). Transfecting cells with $200 \mathrm{MOI}$ of Ad5CMV- $\alpha$ VEGF reduced the amount of VEGF protein to $62.35 \pm 8.24 \mathrm{pg} \mathrm{ml}^{-1} 10^{5}$ cells ${ }^{-1} 24 \mathrm{~h}^{-1}$

Despite the reduction in endogenous VEGF protein secretion by Ad5CMV- $\alpha$ VEGF-infected MDA231-MB cells, the growth rate of these cells was not different from that of mock-or Ad5(d1312)infected MDA231-MB cells (Figure 4).

\section{Xenograft tumour growth after treatment with Ad5CMV- $\alpha$ VEGF}

To determine the effectiveness of Ad5CMV- $\alpha$ VEGF therapy in vivo, we treated mice that had been implanted with MDA231-MB cells with periodic intratumoral injections of Ad5(dl312) or Ad5CMV$\alpha$ VEGF (8 mice/group). 4 months after the treatment began ( 2 months after the final injection), the mean tumour size in the Ad5(d1312) treatment group was $335.23 \pm 83.98 \mathrm{~mm}^{3}$; mean tumour size in the Ad5CMV- $\alpha$ VEGF treatment group was $67.85 \pm 34.65$ $\mathrm{mm}^{3}(P=0.0005)$ (Figure 5). In addition to this direct evidence of an antitumor effect, Ad5CMV- $\alpha$ VEGF produced no adverse effects on gross observation of the health and behaviour of the mice.

\section{VEGF expression and microvessel count in tumours}

Immunohistochemical analysis of tumour tissue that had been treated with Ad5(d1312) showed substantial VEGF protein expression in the diffuse pattern expected of a secreted protein. In contrast, little immunoreactivity was detected in the Ad5CMV$\alpha$ VEGF-treated tumours (Figure 6). To determine whether the reduction in VEGF secretion and the inhibition of tumour growth were associated with a reduction in the ability of the cells to induce neovascularization, we assessed blood vessel density in tumour tissue by immunostaining the specimens with a monoclonal antibody against the endothelial cell surface protein marker CD31. Fewer microvessels were found in tumours treated with Ad5CMV$\alpha$ VEGF $(12.9 \pm 1.85$ per field) than in tumours treated with Ad5 (d1312) $(41.0 \pm 6.72$ per field $)(P=0.03)$.

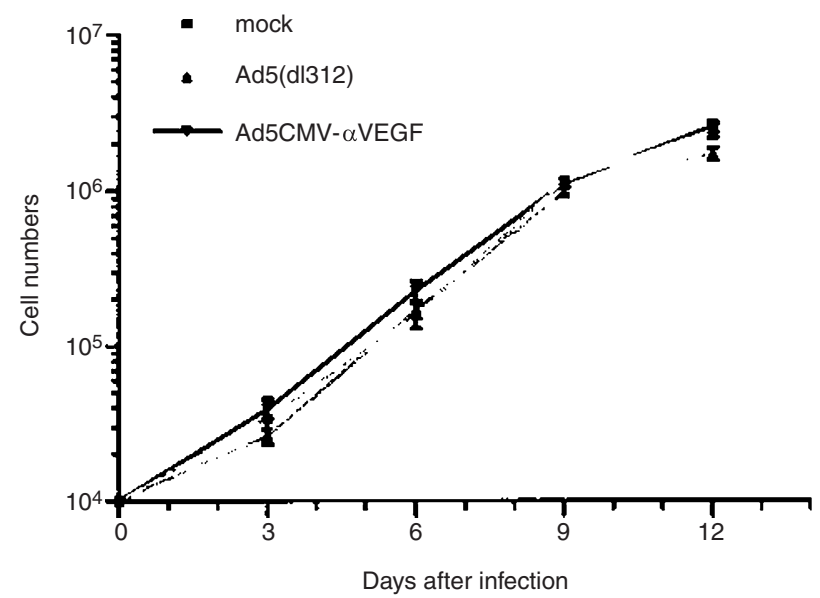

Figure 4 Growth curves for MDA231-MB cells that were mock-infected or infected with $100 \mathrm{MOI}$ of Ad5(d1312) or Ad5CMV- $\alpha$ VEGF. Cells were seeded at a density of $10^{4}$ cells per well and allowed to grow under standard culture conditions for 12 days. The cells were counted using a

haemocytometer every 3 days. The numbers shown are means \pm SD of triplicate dishes

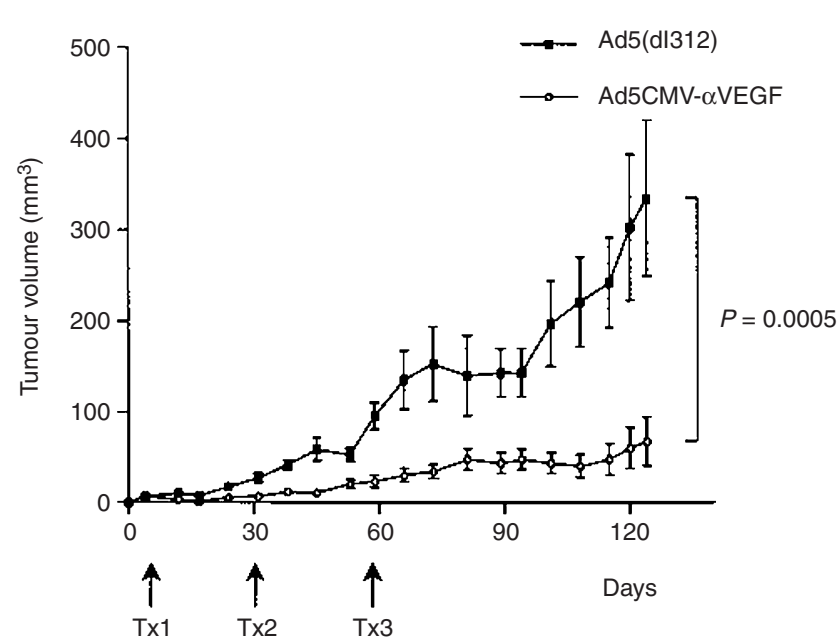

Figure 5 Growth rates of subcutaneous tumours in nude mice treated with intratumoral injections of $5 \times 10^{8}$ PFU Ad5(dl312) $(n=8)$ or Ad5CMV- $\alpha$ VEGF $(n=8)$. Injections were given once every other day over 8-day periods every 4 weeks starting 4 days after tumour-cell implantation. Tumour volumes were calculated from caliper measurements and are presented as the mean \pm SEM $\left(\mathrm{mm}^{3}\right)$ for each group of mice

\section{DISCUSSION}

The purpose of this study was to describe the in vitro and in vivo effect of Ad5CMV- $\alpha$ VEGF, a replication-deficient recombinant adenoviral vector carrying antisense human $\mathrm{VEGF}_{165}$, in breast cancer. Our results showed that the transfer of antisense VEGF cDNA in vitro efficiently down-regulated the secretion of VEGF protein, both in vitro and in an experimental model of breast cancer.

VEGF is a potent endothelial cell mitogen and positive effector of angiogenesis. VEGF is known to be up-regulated in many tumour types (Kim et al, 1993; Asano et al, 1995; Cheng et al, 1996; Saleh et al, 1996; Im et al, 1997). In one study, VEGF RNA expression was significantly elevated in breast cancer specimens relative to that in samples of adjacent non-neoplastic tissue, whereas expression of bFGF, TGF- $\alpha$ and TGF- $\beta$ RNA in tumour tissues was variable (Yoshiji et al, 1996). Up-regulation of VEGF in breast tumour specimens has been correlated with poor prognosis (Weidner et al, 1992; Toi et al, 1995). VEGF was shown in 1 study to be critical for the initial subcutaneous tumour growth of T-47D breast carcinoma cells, whereas other angiogenic factors could compensate for the loss of VEGF after the tumours reached a certain size (Yoshiji et al, 1997).

Our results, and those of others, suggest that targeting the endothelial cells of a tumour with antiangiogenic molecules can be an effective antitumor strategy. In one study, transfecting VEGF cDNA into xenografted C6 rat glioma cells produced hypervascularization of the tumour with abnormally large vessels; the abrupt withdrawal of VEGF resulted in regression of these vessels (Saleh et al, 1996). On the other hand, treating tumours with monoclonal antibodies specific to VEGF decreased or completely inhibited their neovascularization (Kim et al, 1993). Inhibition of endogenous VEGF expression by using an antisense VEGF sequence reduced vascularization and drastically suppressed the tumorigenicity of cancer cell lines (Saleh et al, 1996). We previously found that VEGF could be down-regulated in glioma cells by using ribozymes designed to target the VEGF sequence (Ke et al, 1998). In the present study, Ad5CMV- $\alpha$ VEGF did not affect the growth of the 

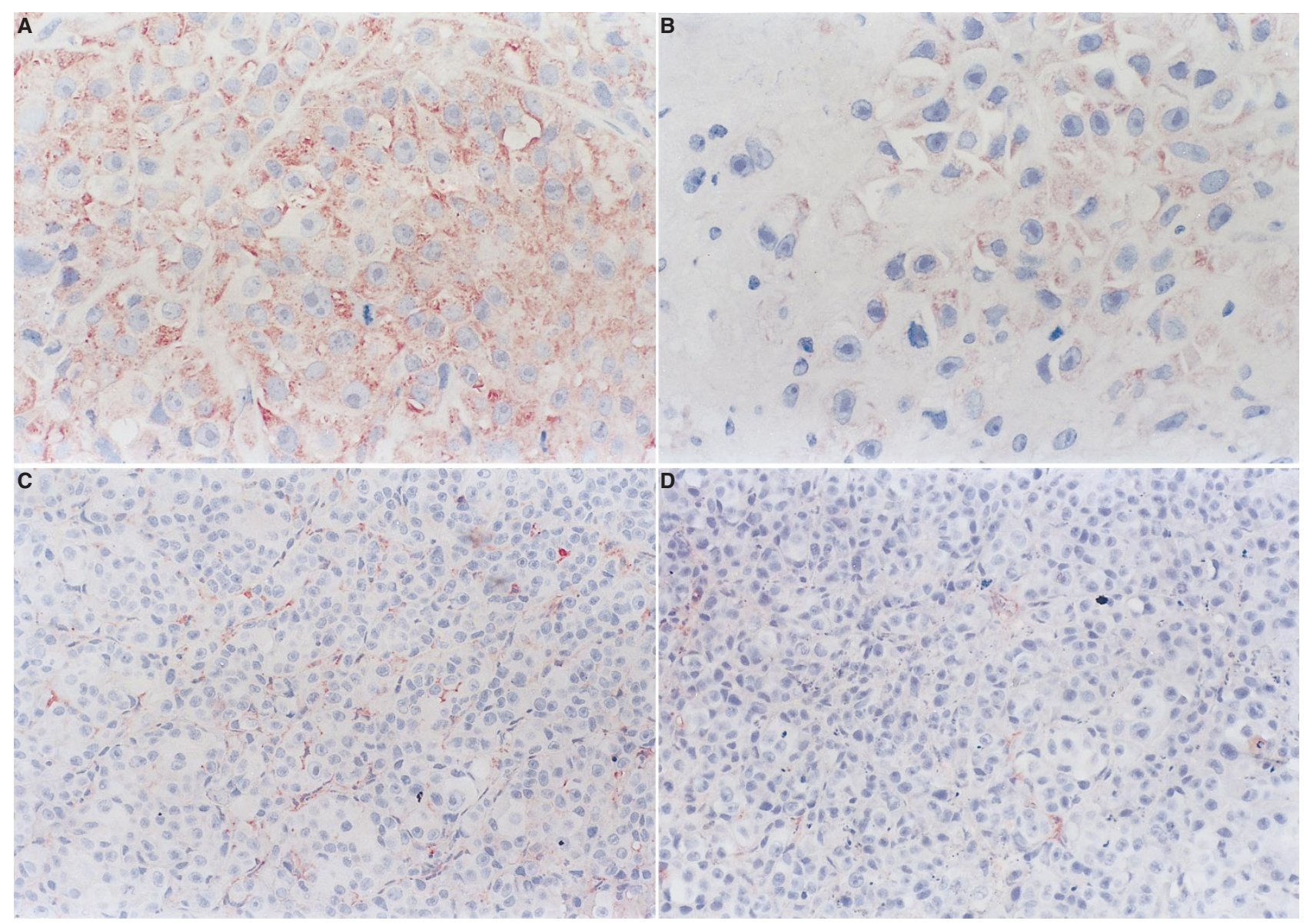

Figure 6 Immunohistochemical staining of mammary fat pad xenografts of MDA231-MB cells with anti-VEGF antibody (A,B) or CD31 monoclonal antibody $(\mathbf{C , D})$ after treatment with Ad5(d1312) (A,C) or Ad5CMV- $\alpha$ VEGF (B,D). VEGF expression, indicated by the intensity of brownish granules in the cytoplasm, was lower in the Ad5CMV- $\alpha$ VEGF-treated tumour (B) than in the Ad5(d1312)-treated tumour (A) (400x). Similarly, the number of brown-staining microvessels were much less dense in the Ad5CMV- $\alpha$ VEGF-treated tumour (D) than in the Ad5(d1312)-treated tumour (C) (200x)

tumour cells in vitro, but significantly suppressed the growth of tumour xenografts and reduced the levels of VEGF protein in vivo, a finding consistent with the apparent anticancer effects of other angiogenesis inhibitors (Beohm et al, 1997; Tanaka et al, 1998).

The results of our work suggest that anti-sense therapy targeting VEGF may have clinical implications for breast cancer treatment. However, several issues remain to be addressed, and some of them cannot be examined using currently available animal models. The host range of human adenoviruses is restricted, and no appropriate animal models exist to allow further exploration of the effectiveness of adenoviral vectors in immune-competent animal models. In a realistic scenario, intratumoral administration of the adenovirus in cancer patients, particularly after multiple treatments, may result in the production of neutralizing antibodies with subsequent elimination of infected cells. However, additional virus-induced cytotoxic effect might be beneficial. Thus, an immune response directed against viral antigens might augment tumour killing by affecting non-infected tumour cells. In addition, the intratumoral recruitment and stimulation of tumour-specific T lymphocytes theoretically could lead, in some cases, to systemic anti-tumor immunity.

In summary, we found adenovirus-mediated transfer of an antisense-VEGF gene to be an efficient means of delivering the gene to breast cancer cells in vitro and in vivo. Moreover, this successful delivery of the gene effectively decreased endogenous VEGF levels and suppressed the growth of tumours derived from human breast cancer MDA231-MB cells. These findings underscore the pivotal role of the VEGF system in breast tumour angiogenesis, and suggest that the Ad5CMV- $\alpha$ VEGF vector may be a useful tool for therapeutic targeting of the tumour vasculature in breast cancer.

\section{ACKNOWLEDGEMENTS}

This study was supported in part by a grant from Ewha Womans University Medical College Graduates' Alumnae Association Research Fund, Korea and by US National Institutes of Health grants CA-51148 and CA-55261. We thank Polly S Lee and Mary $\mathrm{T}$ Wang for helpful discussion and purification of the viruses, Louis Hau and Christine Wogan for editorial assistance.

\section{REFERENCES}

Asano M, Yukita A, Matsumoto T, Kondo S and Suzuki H (1995) Inhibition of tumor growth and metastasis by an immunoneutralizing monoclonal antibody to human vascular endothelial growth factor/vascular permeability factor 121 . Cancer Res 55: 5296-5301 
Attia MA and Weiss DW (1966) Immunology of spontaneous mammary carcinomas in mice. V: acquired tumor resistance and enhancement in strain of mice infected with mammary tumor virus. Cancer Res 26: 1787-1800

Boehm T, Folkman J, Browder T and O'Reilly MS (1996) Antiangiogeneic therapy of experimental cancer does not induce acquired drug resistance. Nature 390: 404-407

Blood CH and Zetter BR (1990) Tumor interactions with the vasculature: angiogenesis and tumor metastasis. Biochem Biophys Acta 1032: 89-118

Cheng S, Su Huang H, Nagane M, Ji X, Wang D, Shih CC, Arap W, Huang C and Cavenee WK (1996) Suppression of glioblastoma angiogenicity and tumorigenicity by inhibition of endogeneous expression of vascular endothelia growth factor. Proc Natl Acad Sci USA 93: 8502-8507

Dvorak HF, Detmar M, Claffey KP, Nagy JA, Van De Water L and Senger DR (1995) Vascular permeability factor/vascular endothelial growth factor: an important mediator of angiogenesis in malignancy and inflammation. Int Arch Allergy Appl Immunol 107: 233-235

Folkman J (1994) Angiogenesis and breast cancer. J Clin Oncol 12: 441-443

Gomez-Manzano C, Fueyo J, Kyritsis AP, Steck PA, Roth JA, McDonnell TJ, Steck KD, Levin VA and Yung WKA (1996) Adenovirus- mediated transfer of the p53 gene produces rapid and generalized death of human glioma cells via apoptosis. Cancer Res 56: 694-699

Graham FL and Prevec L (1991) Manipulation of adenovirus vectors. In: Murray EJ (ed) Gene Transfer and Expression Protocols (Methods in Molecular Biology, Vol. 7), pp 109-128. Human Press, Inc: Clifton NJ

Im SA, Lee SN and Kim SS (1997) Tumor angiogenesis as a predictor of prognosis in gastric carcinoma. J Korean Cancer Assoc 29: 640-647

Im SA, Gomez-Manzano C, Fueyo J, Liu TJ, Ke LD, Kim JS, Lee HY, Steck PA, Kyritsis AP and Yung WKA (1999) Antiangiogenesis treatment for gliomas: transfer of antisense-vascular endothelial growth factor inhibits tumor growth in vivo. Cancer Res 59: 895-900

Jones N and Shenk T (1979) An adenovirus type 5 early gene function regulates expression of other early viral genes. Proc Natl Acad Sci USA 76: 3665-3669

Ke LD, Fueyo J, Chen X, Steck PA, Shi YX, Im SA and Yung WKA (1998) A novel approach to glioma gene therapy: down-regulation of the vascular endothelial growth factor in glioma cells using ribozymes. Int J Oncol 12: 1391-1396
Kim KJ, Li B, Winer J, Armanini M, Gillett N, Phillips HS and Ferrara N (1993) Inhibition of vascular endothelial growth factor-induced angiogenesis suppresses tumor growth in vivo. Nature 362: 841-844

Saleh M, Stacker SA and Wilks AF (1996) Inhibition of growth of C6 glioma cells in vivo by expression of antisense vascular endothelial growth factor sequence. Cancer Res 56: 393-340

Shibuya M (1995) Role of VEGF-Flt receptor system in normal and tumor angiogenesis. Adv Cancer Res 67: 281-316

Takahashi Y, Kitadai Y, Bucana CD, Cleary KR and Ellis LM (1995) Expression of vascular endothelial growth factor and its receptor, KDR, correlates with vascularity, metastasis, and proliferation of human colon cancer. Cancer Res 55: 3964-3968

Tanaka T, Cao Y, Folkman J and Fine HA (1998) Viral vector- targeted antigangiogenic gene therapy utilizing an angiostatin complementary DNA. Cancer Res 58: 3362-3369

Toi M, Inada K, Suzuki H and Tominaga T (1995) Tumor angiogenesis in breast cancer: its importance as a prognostic indicator and the association with vascular endothelial growth factor expression. Breast Cancer Res Treat 36: 193-204

Weidner N, Folkman J, Pozza F, Bevilacqua P, Allred EN, Moore DH, Meli S and Gasparini G (1992) Tumor angiogenesis: a new significant and independent prognostic indicator in early stage breast carcinoma. J Natl Cancer Inst $\mathbf{8 4}$ : 1875-1887

Yoshiji H, Gomez DE, Shibuya M and Thorgeirsson UP (1996) Expression of vascular endothelial growth factor, its receptor, and other angiogenic factor in human breast cancer. Cancer Res 56: 2013-2016

Yoshiji H, Harris SR and Thorgeirsson UP (1997) Vascular endothelial growth factor is essential for initial but not continued in vivo growth of human breast carcinoma cells. Cancer Res 57: 3924-3928

Zhang WW, Fang X, Branch CD, Mazur W, French BA and Roth JA (1993) Generation and identification of recombinant adenovirus by liposome-mediated transfection and PCR analysis. Biotechniques 15: $868-872$ 